Article

\title{
Laccase Activity as an Essential Factor in the Oligomerization of Rutin
}

\author{
Abel Muñiz-Mouro ${ }^{(D)}$, Beatriz Gullón, Thelmo A. Lú-Chau ${ }^{\mathbb{D}}$, María Teresa Moreira, \\ Juan M. Lema and Gemma Eibes * (D) \\ Department of Chemical Engineering, Institute of Technology, Universidade de Santiago de Compostela, \\ 15782 Santiago de Compostela, Spain; abel.muniz@usc.es (A.M.-M.); beatriz.gullon@usc.es (B.G.); \\ thelmo.lu@usc.es (T.A.L.-C.); maite.moreira@usc.es (M.T.M.); juan.lema@usc.es (J.M.L.) \\ * Correspondence: gemma.eibes@usc.es; Tel.: +34-89-8181-6016; Fax: +34-88-1816-702
}

Received: 9 July 2018; Accepted: 3 August 2018; Published: 6 August 2018

\begin{abstract}
The enzyme-mediated polymerization of bioactive phenolic compounds, such as the flavonoid rutin, has gained interest due to the enhanced physico-chemical and biological properties of the products, which increases their potential application as a nutraceutical. In this work, the influence of enzyme activity on rutin oligomerization was evaluated in reactions with low (1000 U/L) and high (10,000 U/L) initial laccase activities. For both reactions, high molecular weight oligomer fractions showed better properties compared to lower weight oligomers. Products of the reaction with low laccase activity exhibited thermal stability and antioxidant potential similar to control reaction, but led to higher inhibitory activity of xanthine oxidase and apparent aqueous solubility. Oligomers obtained in the reaction with high laccase activity showed better apparent aqueous solubility but decreased biological activities and stability. Their low antioxidant activity was correlated with a decreased phenolic content, which could be attributed to the formation of several bonds between rutin molecules.
\end{abstract}

Keywords: rutin oligomers; laccase activity; aqueous solubility; antioxidant activity; xanthine oxidase inhibition; MALDI-TOF; HPSEC

\section{Introduction}

Flavonoids are one of the main types of polyphenols commonly identified in plants as secondary metabolites [1]. This type of compound exhibits satisfactory antioxidant capacities [2,3] and pharmacological properties, with potential use in the prevention of various diseases such as diabetes, cancer, cardiovascular, and neurodegenerative diseases [4,5]. Rutin (3',4',5,7-tetrahydroxy-flavone-3-rutinoside) is a quercetin O-glycoside generally extracted from Fagopyrum esculentum M. (Polygonaceae), Ruta graveolens L. (Rutaceae), Sophora japonica L. (Fabaceae), and Eucalyptus spp. (Myrtaceae). Concerning food, rutin can be found in vegetables, fruits, and plant-derived beverages. Rutin-rich foods had already been used by traditional Chinese medicine [6], and more recently rutin has been demonstrated to possess beneficial properties for preventing diseases and protecting genome stability [7]. Due to its useful properties, the Dietary Supplement Label Database lists over 1100 currently marketed products that contain rutin [8]. However, both its low solubility in aqueous and non-toxic organic solvents, which imply scarce bioavailability, and poor thermal stability [9-11], restrict its application. Enzymatic polymerization of polyphenolic monomers has been shown to produce higher molecular weight polyphenols with improved solubility, thermostability, and superior antioxidant properties [12-16].

Laccases (EC 1.10.3.2) and peroxidases (EC 1.11.1) are enzymes that oxidize phenolic substrates to form phenoxyl radicals. The pathway followed by these radicals depends on various factors such as the nature of the substrate or the composition of the reaction medium. One of the possibilities 
relies on an oxidative coupling that can lead to the formation of oligomers/polymers from the initial substrate [17]. The main advantage of laccase compared to peroxidases is that it requires oxygen as a final electron acceptor rather than hydrogen peroxide [18]. Laccases have been used in the food, pharmaceutical, and cosmetics sectors and have proved their usefulness as catalysts for the oligomerization or polymerization of phenolic compounds [14-16].

More specifically, the production of oligomers from flavonoids with improved properties using laccase has been demonstrated. Kurisawa et al. [14] reported the oligomerization of rutin by laccase, obtaining products with higher superoxide scavenging activity and aqueous solubility compared with the rutin monomer. Moreover, the improved capacity of rutin oligomers to inhibit the xanthine oxidase enzyme [19] and their improved antigenotoxic activity [20] compared to the rutin monomer were also demonstrated.

Although the properties of oligorutin are of great interest, reaction conditions should be optimized to make the process more efficient, both economically and environmentally. Methanol is often used as a co-solvent to allow for higher rutin solubility, but because of its toxicity, it would be advisable to avoid its use, which may involve lower costs in subsequent purification of the products, as methanol cannot be present especially in cosmetics and food applications. Ethanol is a valid alternative organic solvent as it increases the solubility of rutin and is considered safe according to the European Food Safety Authority (EFSA).

The aim of this work is to evaluate the effect of laccase activity on the rutin oligomers produced in a reaction medium compatible with food applications, using ethanol as co-solvent. To our knowledge, there are no studies that comprehensively evaluate the influence of laccase activity on the oligomerization reaction. The products obtained were divided into fractions of different molecular masses by ultrafiltration and subsequently characterized in terms of chemical structure (matrix-assisted laser desorption/ionization-time-of-flight mass spectrometry (MALDI-TOF) and high pressure size exclusion chromatography (HPSEC)), phenolic content, antioxidant activity by different assays (Ferric reducing antioxidant power-FRAP, cupric reducing antioxidant capacity-CUPRAC, 2,2'-azino-bis(3-ethylbenzenothiazoline-6-sulfonic acid) diammonium salt scavenging activity-ABTS ${ }^{+}$ and xanthine oxidase inhibition) and aqueous solubility. The thermal stability of oligorutins produced in both reactions was also assessed by thermogravimetric analysis (TGA).

\section{Results and Discussion}

\subsection{Polymerization Reaction}

The use of EtOH as a co-solvent greatly increased rutin solubility. As shown in Figure S1, rutin solubility increased from $97.1 \mathrm{mg} / \mathrm{L}(0 \% \mathrm{EtOH})$ up to $3370.1 \mathrm{mg} / \mathrm{L}(50 \% \mathrm{EtOH})$. Combining this result with previous studies on laccase stability in the presence of $\mathrm{EtOH}$ [15], the value of $50 \% \mathrm{EtOH}$ : acetate buffer $(\mathrm{pH} 5,10 \mathrm{mM})$ was chosen as the appropriate reaction medium for this work.

Color changes were observed during the experiments, being more gradual in reaction $\mathrm{A}$ than in reaction $B$. Reaction A started to take an orange tone during the first few hours of reaction, eventually turning into an amber-colored liquid. Experiment B turned dark brown in less than an hour and continued to darken for the next hour, when visual changes could not be seen for the remaining extent of the reaction. This change in color is in accordance with that found by Sun et al. [21] for the polymerization of catechol and resorcinol by laccase. In contrast, controls maintained a pale-yellow color during the entire experiments. The enzymatic activity and the rutin concentration in the reaction medium over time are showed in Figure 1 (measurements related to their initial value).

Both the greatest loss of enzymatic activity and the highest rutin conversion occurred during the first 5-6 hours of reaction in both experiments. At that time, the losses in laccase activity reached values close to $50 \%$ of the initial activity, which is consistent with the decrease due to the presence of ethanol in the reaction medium, as previously reported [15]. Nevertheless, during the first two hours, the decrease in laccase activity in reaction B was lower than that observed in reaction A (14\% and 33\%, 
respectively). The enzyme activity continued to decrease until the experiments were completed, with a residual activity of $34 \%$ for reaction A and only $13 \%$ for reaction $\mathrm{B}$.

The increase in laccase activity from 1000 to 10,000 U/L led to a higher rutin throughput (above 93\%). Rutin depletion in reaction A occurred more gradually than in reaction B. After the first five hours, only $42.6 \%$ of rutin was consumed in reaction A, whilst reaction B showed a rapid drop in rutin content leaving only $7 \%$ of the initial rutin unreacted. Similar yields were obtained by Kurisawa et al. [14] using methanol as co-solvent. In addition, reaction B underwent a slightly higher acidification of the reaction medium. The final $\mathrm{pH}$ of reaction $\mathrm{B}$ was 5.80 , while the $\mathrm{pH}$ of reaction $\mathrm{A}(6.12)$ was almost identical to that of the controls (6.18).
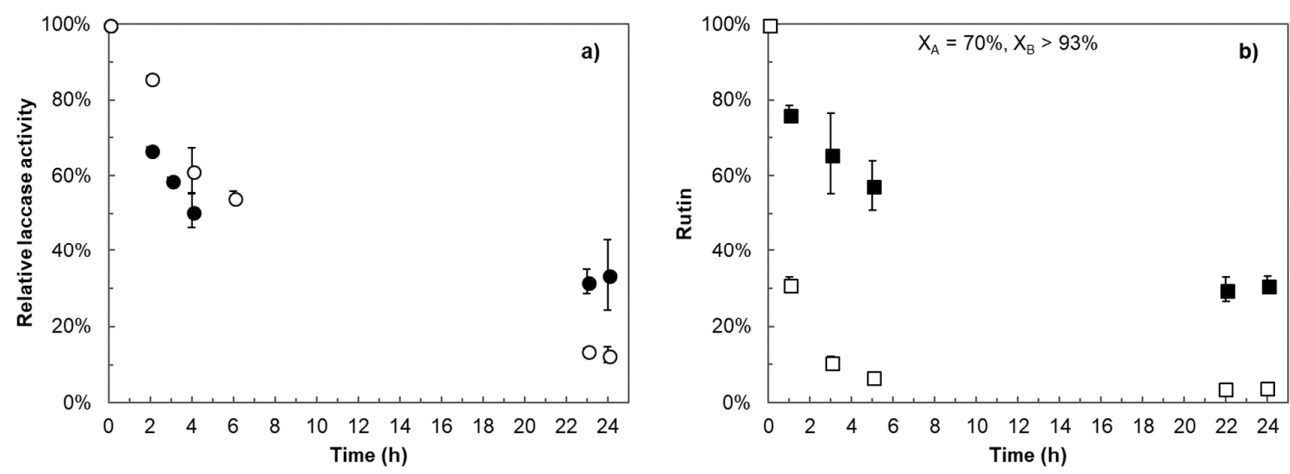

Figure 1. Monitored parameters for the polymerization of $3 \mathrm{~g} / \mathrm{L}$ rutin in $50 \%$ ethanol:acetate buffer (10 mM, pH 5) mixture over $24 \mathrm{~h}$ at $25^{\circ} \mathrm{C}$ and $1500 \mathrm{rpm}$ for reactions $\mathrm{A}$ (1000 U/L of laccase activity) and B (10,000 U/L): (a) relative enzymatic activity of reactions A $(\bullet)$ and B $(\bigcirc)$, (b) residual rutin percentage in the reaction medium for reactions $A(\square)$ and $B(\square)$. X is used to indicate rutin conversion into oligomers.

Both media were divided into three different fractions by ultrafiltration. Although the samples were filtered to reach the same volume for each fraction, once lyophilized, different amounts of solid products were obtained, thus indicating different oligomerization degrees.

\subsection{Apparent Solubility of Rutin Oligomers}

The apparent solubility of the different fractions of the oligomers obtained in reactions A and B was evaluated. The total flavonoid content (TFC) of sample solutions in both $50 \%$ methanol and water supernatants was determined as mg of rutin equivalents per gram of sample (Table S1) and the apparent aqueous solubility was calculated using Equation (1). The results are shown in Table 1.

Table 1. Apparent solubility of oligomer fractions obtained in reactions A and B. The fold is expressed as the improvement of the solubility of the fractions by taking the controls as a reference.

\begin{tabular}{ccc}
\hline \multirow{2}{*}{ Fraction } & Apparent Solubility & Fold \\
\cline { 2 - 3 } & $\mathrm{g} / \mathbf{L}$ & Relative to Controls \\
\hline AF3 & $6.76 \pm 0.04$ & $\sim 58$ \\
AF2 & $5.84 \pm 0.40$ & $\sim 50$ \\
AF1 & $2.21 \pm 0.43$ & $\sim 19$ \\
CA & $0.12 \pm 0.01$ & \\
BF3 & $10.77 \pm 0.21$ & $\sim 71$ \\
BF2 & $9.93 \pm 0.28$ & $\sim 65$ \\
BF1 & $*$ & $*$ \\
CB & $0.15 \pm 0.01$ & \\
\hline
\end{tabular}

* Recovered lyophilized product was not sufficient to perform the assay. (A: $1000 \mathrm{U} / \mathrm{L}$ laccase, B: 10,000 U/L laccase, C: Control, F3 $\geq 10 \mathrm{kDa}, \mathrm{F} 2 \subset(10,1] \mathrm{kDa}, \mathrm{F} 1<1 \mathrm{kDa})$. 
The apparent aqueous solubility of CA and CB controls was similar to that obtained for rutin by Krewson et al. [9], thus supporting the validity of the technique used to indirectly assess the solubility of these flavonoids. The results showed that, regardless of the laccase activity used, an increase in the molecular mass of the oligomers led to an improved apparent aqueous solubility, as previously reported for different flavonoid oligomers $[16,19,20]$. Moreover, higher enzymatic activity during rutin polymerization (reaction B) produced oligomers (F3 fractions) with considerably enhanced apparent solubility, 70 times greater than its control. Nevertheless, the use of $1000 \mathrm{U} / \mathrm{L}$ laccase activity (reaction A) improved the apparent solubility nearly 60 times, which would imply lower production costs.

A molecular modeling study conducted by Anthoni et al. [19] indicated that higher solubility of oligorutins could be attributed to their unfolded molecular structure, which would allow a greater number of intermolecular H-bonds of sugar parts with water molecules.

\subsection{Antioxidant Activities of Rutin Oligomer Fractions and Xanthine Oxidase Inhibitory Potential}

Oligorutins previously obtained by different researchers in methanol: water mixtures have shown good scavenging and antioxidant activity [14]. In addition, their ability to inhibit the enzyme xanthine oxidase, involved in inflammatory-related processes, has also been proven [19]. To evaluate the antioxidant activity of the lyophilized oligorutin fractions produced in reactions A and B, FRAP and CUPRAC methods were used. Moreover, the ABTS assay allowed us to measure their radical scavenging capacity. The results are shown in Table 2.

Table 2. Ferric reducing antioxidant power (FRAP), cupric reducing antioxidant capacity (CUPRAC), $\mathrm{ABTS}^{+}$scavenging activity and xanthine oxidase inhibitory potential of lyophilized rutin oligomer fractions and their respective controls for the different reaction conditions.

\begin{tabular}{ccccc}
\hline \multirow{2}{*}{ Fraction } & FRAP & CUPRAC & ABTS $^{+}$ & Xanthine Oxidase Inhibition \\
\cline { 2 - 5 } & mg TE ${ }^{\text {/g Sample }}$ & mg TE $^{\text {a }}$ /g Sample & \% Inhibition $\mathbf{( 1 ~} \mathbf{~ / L ) ~}$ & IC $_{\mathbf{5 0}}$ (mg/L) \\
\hline AF3 & $149.27 \pm 22.67$ & $488.30 \pm 2.48$ & $98 \pm 1$ & $186.36 \pm 18.28$ \\
AF2 & $138.02 \pm 3.55$ & $415.14 \pm 7.43$ & $95 \pm 1$ & $198.43 \pm 14.90$ \\
AF1 & $81.87 \pm 1.93$ & $245.67 \pm 12.11$ & $71 \pm 2$ & $372.30 \pm 30.47$ \\
CA & $168.79 \pm 2.58$ & $526.63 \pm 8.81$ & $100 \pm 1$ & $259.84 \pm 1.45$ \\
BF3 & $59.22 \pm 1.29$ & $226.21 \pm 22.56$ & $60 \pm 3$ & $241.28 \pm 24.34$ \\
BF2 & $13.33 \pm 0.43$ & $46.08 \pm 3.44$ & $11 \pm 1$ & $>400^{\mathrm{b}}$ \\
BF1 & $12.11 \pm 2.79$ & $27.50 \pm 1.10$ & $8 \pm 1$ & $>600^{\mathrm{b}}$ \\
CB & $109.37 \pm 2.79$ & $304.24 \pm 65.76$ & $85 \pm 3$ & $415.12 \pm 2.72$ \\
\hline
\end{tabular}

A: 1000 U/L laccase, B: 10,000 U/L laccase, C: control, F3 $\geq 10 \mathrm{kDa}, \mathrm{F} 2 \subset(10,1] \mathrm{kDa}, \mathrm{F} 1<1 \mathrm{kDa} .{ }^{\text {a }} \mathrm{TE}=$ Trolox equivalents. ${ }^{b}$ maximal tested concentration due to the impossibility of measuring higher absorbance.

The differences observed in both control reactions, $\mathrm{CA}$ and $\mathrm{CB}$, derive from their different content of inactivated enzyme (since reaction $B$ had 10-fold higher enzymatic activity, inactivated laccase represents a higher mass percentage of the final freeze-dried powder obtained). Experiment $B$ involved 10 times the enzyme activity in A, thus, after lyophilization, the non-rutin content in medium B was higher than in medium A, causing its lower antioxidant activity.

For all fractions, the antioxidant activity was lower than that of their control. AF3 showed the values most similar to those of the controls, reaching about $90 \%$ of the CA antioxidant activity for FRAP and CUPRAC methods and almost $100 \%$ of its $\mathrm{ABTS}^{+}$radical scavenging capacity. The use of 10,000 U/L laccase activity caused a remarkable loss in antioxidant activity, with the BF3 fraction reaching values close to $70 \%$ of those obtained for CB (except for the FRAP assay, which showed only $54 \%$ ). Antioxidant activity dropped for smaller Mw fractions, especially for BF2 and BF1 oligomers, where close to $10-15 \%$ of $C B$ activities were found, while AF2 and AF1 retained $\sim 80$ and $~ 50 \%$, respectively (except for the ABTS test, which showed 95 and $71 \%$ of the CA scavenging activity).

This loss in antioxidant activity did not match the results provided by Kurisawa et al. [14], who observed an improvement in the superoxide scavenging capacity of oligorutin compared to rutin. However, Anthoni et al. [19] did observe this drop in antioxidant activity and, as stated by several 
authors who studied the effect of phenolic substituents on antioxidant activity [22,23], attributed it to a possible loss of free hydroxyl groups on $\mathrm{C}^{\prime}$ ' and / or C $\mathrm{C}^{\prime}$. These functional groups, together with the $\mathrm{C} 2-\mathrm{C} 3$ double bond, are the structural features in rutin molecule that contribute the most to its antioxidant activity (the chemical structure of rutin can be seen in Figure S2). Therefore, the phenolic groups in C4' and C3' could have taken part in the polymerization reaction, leading to the possible formation of ether and/or carbon-carbon linkages between different rutin molecules.

The enzyme xanthine oxidase is considered to be an important biological source of ROS and catalyzes the oxidation of hypoxanthine and xanthine to uric acid, which plays a crucial role in gout [24]. Xanthine oxidase inhibitors represent an attractive option for the treatment of disorders such as gout, hyperuricemia, ulcers, ischemia, and hypertension, among others [25].

Table 2 shows also the xanthine oxidase inhibitory activity in the oligorutin fractions and their respective controls. The $\mathrm{IC}_{50}$ values are the average result of the duplicates, calculated by standard curve regression analysis. The correlation factors $\left(R^{2}\right)$ for each standard curve ranged from 0.964 to 1000. AF3, AF2 and BF3 showed better results than $\mathrm{CA}$ and $\mathrm{CB}$, with the $\mathrm{IC}_{50}$ of $\mathrm{AF} 3$ being the lowest value $(186 \mathrm{mg} / \mathrm{L})$. This enhancement was also found for AF2 with a similar $\mathrm{IC}_{50}$ but not for AF1, BF2 and BF1, leading to the conclusion that smaller oligomers show the worst xanthine oxidase inhibitory capacity. In fact, for oligomers obtained using higher laccase activity, only BF3 exhibited a lower $\mathrm{IC}_{50}$ than its control. This mass-related inhibitory activity is consistent with previous results reported by Anthoni et al. [19], who found that the higher the molecular mass of the oligorutin fractions, the lower the $\mathrm{IC}_{50}$ is. However, this is not in agreement with the results obtained by Kurisawa et al. [14], who performed rutin oligomerization in methanol:buffer mixtures using Myceliophthora laccase as a biocatalyst.

When comparing the best absolute $\mathrm{IC}_{50}$ values, $\mathrm{AF} 3$ and $\mathrm{AF} 2$ obtained better results than $\mathrm{BF} 3$, which means that the excess of laccase activity used in the production of higher molecular mass oligorutin negatively affected the xanthine oxidase inhibitory activity of these fractions.

The structure-activity relationship of flavonoids as inhibitors of xanthine oxidase has been previously studied by several researchers [24,26], who observed that the aromatic hydroxyl groups placed at $\mathrm{C}^{\prime}$ ' and $\mathrm{C4}^{\prime}$ ' had little or no effect upon this capacity. In contrast, the $\mathrm{OH}$ groups linked to $\mathrm{C} 5$ and $\mathrm{C} 7$, along with the $\mathrm{C} 2-\mathrm{C} 3$ double bond, were the main causes of their xanthine oxidase inhibitory potential. Thus, the phenolic groups in $\mathrm{C} 5$ and $\mathrm{C} 7$ should not have been lost by providing a link between the rutin monomers.

In general, the results of the antioxidant activity tests and the xanthine oxidase inhibitory potential assay indicate that oligomerization probably involves phenolic groups in $\mathrm{C}^{\prime}$ and/or $\mathrm{C} 4^{\prime}$ carbons to form $\mathrm{O}-\mathrm{C}$ or $\mathrm{C}-\mathrm{C}$ bonds.

\subsection{Evaluation of the Phenolic Content of Rutin Polymerization Products}

Since there are widely studied correlations between the phenolic groups of flavonoids and their effect upon antioxidant activity [23] and the xanthine oxidase inhibition potential [24], the phenolic content of the oligorutin fractions produced was measured using two different protocols, revealing different results (Figure 2) that could be attributed to the chemical basis of the methods. While the Folin-Ciocalteu method measures all the reducing phenolic compounds, the 4-aminoantipyrine (4-AAP) method can only detect phenolic groups that have a free para position [27]. Due to the scarce quantity of recovered products for the lower molecular weight fractions, these assays were only performed for the F3 and F2 fractions of both reaction products.

The results provided by the Folin-Ciocalteu assay showed a slight loss of reducing phenolic compounds (RPC) for AF3 of less than 15\% compared to CA, indicating that O-C bonding could have occurred. In addition, the reducing phenolics decreased considerably for the BF3 fraction, thus indicating a higher degree of polymerization (DP) through phenolic groups or, alternatively, that several bonds involving those hydroxyl groups were formed between rutin monomers. For F2 fractions, the reduction in RPC was greater, especially for BF2, which retained only about $10 \%$ of the 
phenolic groups present in the control. Therefore, the Folin-Ciocalteu assay proves that the use of higher enzyme activity led to a further decrease in reducing phenolic compounds, and that the smaller fractions of both reaction products suffered a marked drop in comparison with higher Mw fractions.

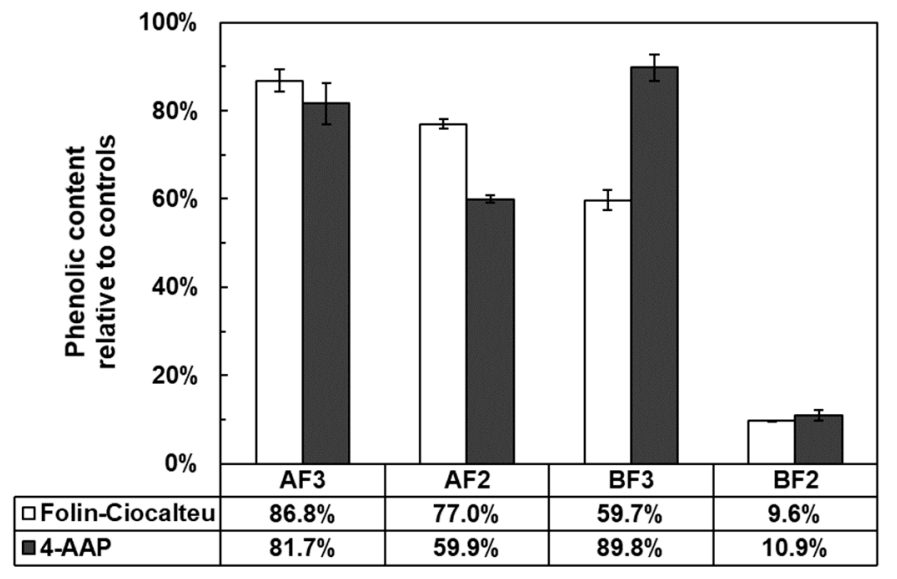

Figure 2. Phenolic content of the different fractions obtained for reaction A and B relative to their respective controls. Values were obtained via Folin-Ciocalteu $(\square)$ and 4-AAP $(\square)$ assays (A: 1000 U/L laccase, B: 10,000 U/L laccase, F3 $\geq 10 \mathrm{kDa}, \mathrm{F} 2 \subset(10,1] \mathrm{kDa})$.

The protocol based on the reaction of phenolic compounds and 4-AAP showed different results. Considering the molecular structure of rutin (Figure S2). it can be understood that this method would not measure hydroxyl groups bonded to $\mathrm{C}^{\prime}$ and $\mathrm{C} 7$, as they do not present a free para position.

The values obtained for the fractions show differences in the decrease of phenolic content that may provide insight on the possible bonding occurring between rutin monomers. While AF3 and BF2 did not show major differences for both protocols used to measure phenolic content, AF2 and BF3 did. AF2 showed a higher decrease of non-para-substituted phenols in proportion to all the reducing hydroxyl groups bonded to the aromatic part of the rutin molecule, while BF3 showed the opposite effect. Therefore, reaction A shows a general tendency to yield oligomers with a lesser proportion of non-para-substituted phenolic groups, while the opposite trend was observed for products of reaction $\mathrm{B}$.

These results lead to the conclusion that the use of higher enzyme activity not only decreased the phenolic content, but also may have affected the phenolic groups involved in the polymerization reaction. However, this information alone does not directly indicate which type of bonding took place between the rutin molecules. A decrease in the phenolic groups detected by the 4-AAP method can mean that the hydroxyl group became part of an $\mathrm{O}-\mathrm{C}$ bond, or that the carbon atom placed in its para position has been involved in a new bond with another rutin molecule ( $\mathrm{C}-\mathrm{O}$ or $\mathrm{C}-\mathrm{C})$.

Furthermore, considering the antioxidant and xanthine oxidase inhibitory activities and phenolic content, the hydroxyl group in C3' appears to be involved in rutin oligomerization when $1000 \mathrm{U} / \mathrm{L}$ of laccase is used, while increasing this activity up to $10,000 \mathrm{U} / \mathrm{L}$ would imply the greater participation of the phenolic group in $\mathrm{C}^{\prime}$ to produce high molecular mass oligomers. This observed trend was indicated in Figure S2, where the atoms marked in blue represent those most likely to be involved in oligomerization under A conditions, whereas those in red would correspond to B conditions.

A similar effect of the enzyme activity has been previously observed on the characteristics of the dehydrogenation polymers (DHP) synthesized from coniferyl alcohol (CA). Mechin et al. [28] demonstrated the influence of enzyme activity/substrate ratio on the structure of DHP's obtained using horseradish peroxidase (HRP). They found a negative correlation between the $\beta-\mathrm{O}-4$ content of the DHPs and the HRP/CA ratio. Hence, the enzyme activity dose had a significant effect on the structure of the obtained products. 
However, if the oligomerization only occurs by $\mathrm{C}-\mathrm{O}$ and/or $\mathrm{C}-\mathrm{C}$ bonds, it is not entirely determined by the techniques and methods already applied. Anthoni et al. [19] performed ${ }^{1} \mathrm{H}-\mathrm{NMR}$ analysis and confirmed the existence of $\mathrm{C}-\mathrm{O}$ and $\mathrm{C}-\mathrm{C}$ bonds between the rutin molecules, not only in the aromatic but also the sugar part. As for the aromatic part, the bonds that might be most likely to form were $\mathrm{C}^{\prime}-\mathrm{C} 2^{\prime}, \mathrm{O}^{\prime}-\mathrm{C} 6^{\prime}$ and $\mathrm{C} 6^{\prime}-\mathrm{C} 6^{\prime}$ [29], the latter two proposed linkages according to our conclusions based on antioxidant and xanthine oxidase inhibitory activities after considering the structure-activity relationships.

\subsection{Molecular Weight Characterization of the Different Reaction Products}

\subsubsection{MALDI-TOF Analysis}

The MALDI-TOF analysis of the obtained fractions (Figure S3) showed different high intensity peaks separated by $\sim 608 \mathrm{Da}$, indicating that two different rutin molecules have lost a hydrogen atom in the formation of a new intermolecular bond.

The highest DP found was 7, only obtained in BF3, thus supporting that the highest laccase activity used in the polymerization reaction implied the longest chain of the oligomers produced, despite the low intensity of the signal. In reaction A, a DP of 6 was found in AF2 and AF3 fractions. This deviation of only one degree of polymerization between AF3 and BF3 would probably not explain the differences in antioxidant and xanthine oxidase inhibitory activities, which might imply that the presence of higher laccase activities may have different effects on the products apart from increasing the length of the oligomer. The results were similar to those obtained by Anthoni et al. [19,30], who found a maximum DP of 6 rutin units using methanol as co-solvent. The intensities read in the AF3 fraction seem to indicate that the R2 and R3 oligomers may be present in the same proportion in this sample. In contrast, BF3 showed a remarkable difference in favor of R3, which coincides with the higher DP displayed by that sample. In addition, all the fractions produced in reaction B show an unidentified compound with a mass close to $1260 \mathrm{Da}$ and intensity similar to that of R2.

The observed masses, intensities and suggested compounds for the peaks found with this technique are listed in Tables S2 and S3, along with the expected theoretical mass of each compound and the deviation of the measured values. Intermediate compounds with lower intensities would correspond to rutin oligomers lacking one or several methyl, $\mathrm{OH}$, glucose, rhamnose, or rutinose substituents. With this in mind, reaction mechanisms similar to those found in esculin oligomerization [15] could be assumed.

The oligomers in reaction B often show the greatest deviations from the theoretical masses of expected polymers. This effect was especially observed for DP greater than 3 and always corresponded to masses lower than expected. The formation of multiple bonds between two rutin molecules in the presence of increased laccase activity would explain this behavior.

\subsubsection{HPSEC Analysis}

High performance size exclusion chromatography (HPSEC) made it possible to measure the average molecular mass of the oligomers present in the different fractions obtained after ultrafiltration (Figure 3). These results are orientative and can only be used in a comparative study, as the calibration curve was carried out using polystyrene standards (266 to $62,500 \mathrm{Da}$ ).

The products of reaction A were well distributed between AF2 and AF3, since high molecular mass compounds were detected in both chromatograms. The highest Mw, registered by BF3, was $8338.51 \mathrm{Da}$, much higher than that observed in the MALDI-TOF analysis, which corresponds to a maximum degree of polymerization of 14 instead of 7 . The same effect but on a smaller scale occurred for all fractions analyzed. The mass underestimation by the MALDI-TOF technique, also reported by Anthoni et al. [30], was attributed to the possibility of a weak ionization for oligomers with a molecular mass greater than $3000 \mathrm{Da}$. 
Compounds of about 4000 and 400 Da were detected in CA and CB, respectively, probably derived from the use of $T$. versicolor laccase. The differences between the chromatograms may be linked to the amount of laccase and rutin present in each control (rutin and laccase HPSEC chromatograms are provided in Figure S4). Moreover, a peak of around 400 Da could also be observed in oligomer fractions and BF2 seems to be enriched in this compound, which shows that the oligomers obtained in reaction $B$ are mainly concentrated in the $\mathrm{BF} 3$ fraction, which would explain the lower antioxidant activities and poor xanthine oxidase inhibitory capacity present in BF2 and BF1. The AF3 and AF2 chromatograms did not differ much, despite being AF3 enriched with oligomers of a slightly higher Mw.
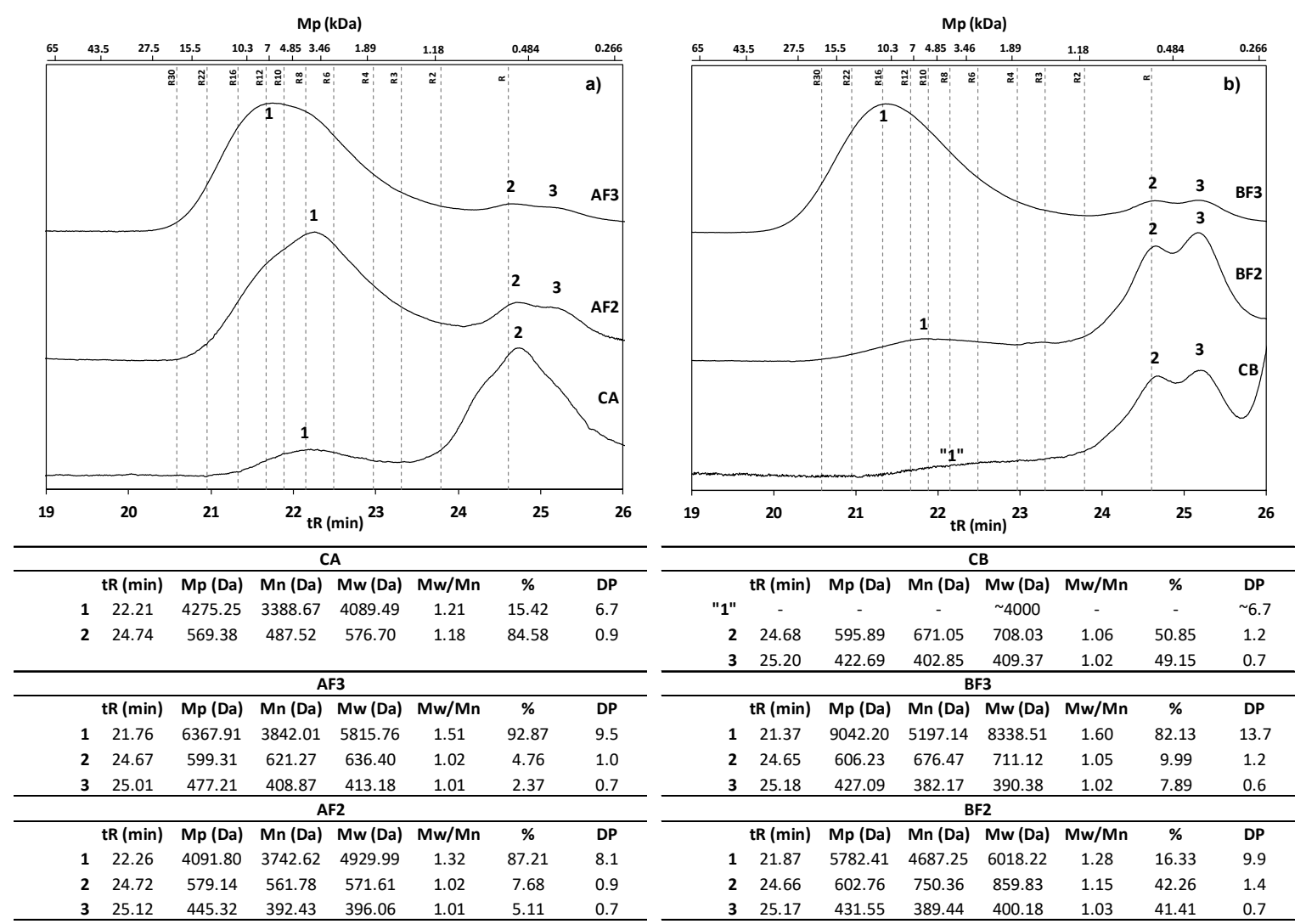

Figure 3. Molecular weight, polydispersity, and degree of polymerization parameter obtained by HPSEC for controls and fractions F3 and F2 of rutin oligomers from reactions A (a) and B (b) (A: $1000 \mathrm{U} / \mathrm{L}$ laccase, B: 10,000 U/L laccase, F3 $\geq 10 \mathrm{kDa}, \mathrm{F} 2 \subset(10,1] \mathrm{kDa})$. tR: retention time, Mp: peak molecular weight, Mn: number-average, Mw: average molecular weight, $\mathrm{Mw} / \mathrm{Mn}$ : degree of polydispersity and DP: degree of polymerization. The percentage of each mean molecular mass peak observed in the quantified area of the chromatogram is also shown in the table.

The differences in the degree of polymerization are, according to several authors, related to the antioxidant properties, but it cannot be stated that the properties evaluated in oligorutins derive only from this aspect, and evidence of possible multiple bonding between rutin molecules should be considered. For this reason, and to check whether this hypothesis is true or false, a new set of experiments was designed, with the objective of producing oligomers of similar molecular mass with 1000 and 10,000 U/L laccase activity.

If multiple linkages have been produced for oligomers in reaction $B$, although the products do not show very different molecular masses, different antioxidant activities are expected. The AF4' and BF5' fractions showed several similarities in the HPSEC chromatograms (see Figure S5) and their antioxidant properties were evaluated by FRAP and CUPRAC assays: BF5' exhibited nearly half of the AF4' antioxidant activity for both assays: $39.29 \pm 3.02$ (FRAP) and $143.44 \pm 2.02 \mathrm{mg}$ TE/g sample 
(CUPRAC) for BF5'; $80.52 \pm 4.06$ (FRAP) and $252.36 \pm 3.17 \mathrm{mg}$ TE/g sample (CUPRAC) for AF4'. Despite both products showing similar DP, these comparisons make evident that different laccase activities used for each reaction have led to structurally different oligorutin.

\subsection{Thermal Stability of Rutin Oligomers}

The thermal stability of high molecular mass oligomer fractions and lyophilized controls for both reactions was assessed by thermogravimetric analysis. The differences in the controls are attributed to their different laccase content, thus decreasing the solid content at $600{ }^{\circ} \mathrm{C}$ for $\mathrm{CB}$, since commercial laccase degrades up to around $85 \%$ at this temperature. AF3 showed almost the same solid residue at $600{ }^{\circ} \mathrm{C}$ as CA, while thermal stability in BF3 was strongly compromised, with only $25.7 \%$ of solid residue at the end of the analysis (final solid residue values along with decomposition intervals are presented in Table S4).

The TGA curves (Figure 4) showed a continuous decrease in mass at low temperatures for both $\mathrm{AF} 3$ and BF3 at the beginning of the analysis. Nevertheless, while the mass loss in AF3 was comparable to $\mathrm{CA}$ and $\mathrm{CB}$ (3-4\% of the total product), BF3 lost up to $8.6 \%$ of its mass. Losses below $\sim 120{ }^{\circ} \mathrm{C}$ are often attributed to the moisture content of the samples [31], but, since all samples were manipulated identically, these observed losses could also be associated with the chemical structure of the oligomers, either if the samples are partially decomposing or their hygroscopicity changed, which would relate this major mass loss for BF3 to a higher amount of ambient humidity absorbed after lyophilization.

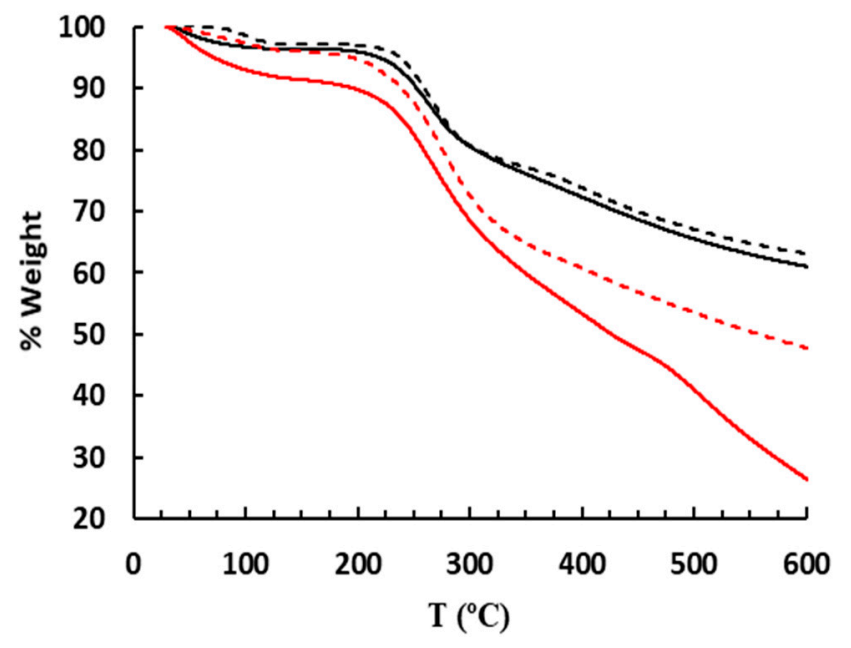

Figure 4. Thermogravimetric analysis (TGA) analysis for high molecular mass rutin oligomers obtained in reactions $\mathrm{A}$ and $\mathrm{B}$, compared to control experiments. - AF3, - - CA, - BF3, - - CB (A: $1000 \mathrm{U} / \mathrm{L}$ laccase, B: 10,000 U/L laccase, C: control-F3 $\geq 10 \mathrm{kDa}$ ).

Unlike AF3, $\mathrm{CA}$ and $\mathrm{CB}, \mathrm{BF} 3$ showed three different decomposition steps (inflection points in the TGA curve) and the solid residue at $600{ }^{\circ} \mathrm{C}$ was lower than that of its control, thus showing poor thermal stability, which cannot be attributed to the use of different laccase activities compared to AF3, but to its chemical nature. The properties of oligorutins evaluated in this study were summarized in Table S5.

\section{Materials and Methods}

\subsection{Materials}

Laccase from Trametes versicolor, rutin hydrate, 2,2'-azino-bis(3-ethylbenzenothiazoline-6-sulfonic acid) diammonium salt (ABTS), potassium persulfate, aluminum chloride hexahydrate, sodium nitrite, iron (III) chloride hexahydrate, 2,4,6-tri(2-pyridyl)-1,3,5-triazine (TPTZ), neocuproine, 
6-hydroxy-2,5,7,8-tetramethylchroman-2-carboxylic acid (Trolox), Folin-Ciocalteu's phenol reagent, 4-aminoantipyrine (4-AAP), potassium ferricyanide, xanthine, xanthine oxidase (E.C. 1.17.3.2.), gallic acid, dimethyl sulfoxide (DMSO), absolute ethanol, methanol and polystyrene standards (between 62,500 and $266 \mathrm{Da}$ ) were purchased from Sigma (Sigma-Aldrich, St. Louis, MO, USA). Copper (II) chloride dihydrate and phenol were purchased from Merck (Merck KGaA, Darmstadt, Germany). Di-sodium hydrogen phosphate anhydrous was purchased from Panreac (Panreac Química SLU, Barcelona, Spain). 2,6-dimethoxyphenol (2,6-DMP) was purchased from Fluka (Honeywell Specialty Chemicals Seelze $\mathrm{GmbH}$, Seelze, Germany). All reagents were of analytical grade.

\subsection{Laccase Activity}

Laccase activity of the enzyme stock solution was determined using ABTS as substrate [32]. During the oligomerization reaction, the substrate was changed to 2,6-DMP instead of ABTS, to avoid the possible reduction or scavenging of $\mathrm{ABTS}^{+}$by antioxidant compounds present in the reaction medium, such as rutin itself or the oligomers produced, since it is well known that this reverse reaction is possible [33]. This measurement was performed following the protocol defined by Wariishi at al. [34] with slight modifications. Briefly, the oxidation rate of $50 \mu \mathrm{L}$ of $20 \mathrm{mM} 2,6-\mathrm{DMP}$ to $2,2^{\prime}, 6,6^{\prime}$-tetramethoxydibenzo-1,1'-diquinone caused by $50 \mu \mathrm{L}$ of laccase solution, was monitored at $468 \mathrm{~nm}\left(\varepsilon_{468}=49,600 \mathrm{M}^{-1} \cdot \mathrm{cm}^{-1}\right)$ in a sodium malonate $(250 \mathrm{mM}, \mathrm{pH} 4.5,200 \mu \mathrm{L})$ and distilled water $(700 \mu \mathrm{L})$ mixture at room temperature. One unit $(\mathrm{U})$ of activity was defined as the amount of enzyme forming $1 \mu \mathrm{mol}$ of 2,2',6,6'-tetramethoxydibenzo-1,1'-diquinone per minute. All spectrophotometric measurements were carried out on a Shimadzu UV-1800 (Shimadzu Europa GmbH, Duisburg, Germany).

\subsection{Synthesis of Rutin Oligomers}

\subsubsection{Oligomerization Reaction}

Polymerization of rutin was performed in $12 \mathrm{~mL}$ polypropylene test tubes with a threaded cap placed inside an MSC-100 thermoshaker incubator. This equipment protected the reaction medium from direct exposure to light. Only $5 \mathrm{~mL}$ of each tube was filled with the reaction medium to ensure oxygen excess (thus not limiting the reaction) and correct agitation. The temperature was set to $25^{\circ} \mathrm{C}$ and agitation to $1500 \mathrm{rpm}$. For both experiments, the initial concentration of rutin was $3 \mathrm{~g} / \mathrm{L}$ and

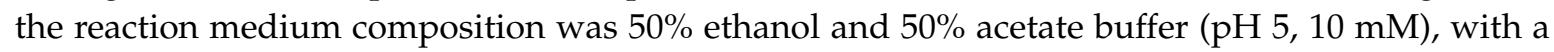
single initial pulse of the enzyme solution to reach the desired initial laccase activity. In Experiment A, $1000 \mathrm{U} / \mathrm{L}$ (ABTS-related units) of laccase was used as a catalyst, whereas 10,000 U/L was used in Experiment B. Control experiments (CA and CB) had exactly the same composition as their reference experiments, but the enzyme was previously thermally inactivated $\left(30 \mathrm{~min}\right.$ at $\left.100{ }^{\circ} \mathrm{C}\right)$. After $24 \mathrm{~h}$, all reactions were stopped by adding $\mathrm{HCl}$ to acidify the reaction medium at a $\mathrm{pH}$ of 1.5.

Rutin concentration was monitored during the oligomerization process by high-performance liquid chromatography (HPLC) in a Jasco XLC HPLC (Jasco Analitica Spain, Madrid, Spain) equipped with a $3110 \mathrm{MD}$ diode array detector (detection at $355 \mathrm{~nm}$ ) and a Gemini reversed-phase column $(150 \times 4.6 \mathrm{~mm}$, particle size: $3 \mu \mathrm{m})$ (Phenomenex, supplied by Jasco Analitica Spain, Madrid, Spain) at $45{ }^{\circ} \mathrm{C}$. Gradient elution (flow rate of $\left.0.7 \mathrm{~mL} \cdot \mathrm{min}^{-1}\right)$ started with $10 \%$ acetonitrile in water $(2 \%$ acetic acid), increased to $90 \%$ acetonitrile in $8 \mathrm{~min}$, and then decreased back to the initial concentration after $2 \mathrm{~min}$.

\subsubsection{Separation and Lyophilization of the Polymers}

After completion of the reaction, the samples were divided into three fractions using an Amicon 8010 10-mL ultrafiltration cell (Millipore Corporation, Bedford, MA, USA), with regenerated cellulose membrane discs with a nominal molecular weight limit of 10 and $1 \mathrm{kDa}$. For each experiment, the fractions F3 (10 kDa retentate), F2 (10 kDa permeate and $1 \mathrm{kDa}$ retentate) and F1 (1 kDa permeate) were obtained and labelled as AF3, AF2, AF1, BF3, BF2 and BF1. Ethanol was removed by evaporation 
from products and controls (Büchi Rotavapor R-205 BÜCHI Labortechnik AG, Flawil, Switzerland, 50-70 mbar, $40^{\circ} \mathrm{C}, 60 \mathrm{rpm}$ ) and samples were then lyophilized (Labconco FreeZone Benchtop Freeze Dry System (Labconco Corporation, Kansas City, MO, USA), 0.098 Torr, $-50{ }^{\circ} \mathrm{C}$ ).

\subsection{Characterization}

\subsubsection{Apparent Solubility of Rutin Oligomers}

Since it was not possible to measure the concentration of rutin oligomers in solution (no standards are available), an indirect protocol for measuring apparent aqueous solubility was developed based on the total flavonoid content (TFC) assay explained by Kim et al. [35] with the modifications proposed by Gullón et al. [36].

With the aim of analyzing the apparent solubility of the target compounds, two different matrices were considered for the preparation of each sample, one in distilled water and one in $50 \%$ methanol. For a concentration of $10 \mathrm{~g} / \mathrm{L}$ of lyophilized fractions and controls, the solution prepared in water showed partial solubilization, which was evidenced by the presence of precipitates and turbidity of the sample, while the solution containing methanol showed complete dissolution. After $1 \mathrm{~h}$ of agitation at $1500 \mathrm{rpm}$ and $25^{\circ} \mathrm{C}$ in an MSC-100 thermoshaker incubator (LABGENE Scientific SA, Châtel-Saint-Denis Switzerland), each pair of samples was centrifuged at $25^{\circ} \mathrm{C}$ and $14,000 \mathrm{rpm}$ using an Eppendorf $5417 \mathrm{R}$ microcentrifuge. The supernatant from each sample was withdrawn and the TFC was measured as described above and expressed as an average of two duplicates. The apparent solubility $(\mathrm{g} / \mathrm{L})$ of the products was then calculated as indicated in Equation (1):

$$
\text { Apparent solubility }=\frac{\mathrm{TFC}_{\mathrm{H}_{2} \mathrm{O}}}{\mathrm{TFC}_{\mathrm{MeOH}: \mathrm{H}_{2} \mathrm{O}}} \cdot \mathrm{C}
$$

where $\mathrm{TFCH}_{2} \mathrm{O}$ is the TFC in the supernatant of aqueous solutions, TFCMeOH: $\mathrm{H}_{2} \mathrm{O}$ is the TFC in methanol:water solutions, and $\mathrm{C}$ is the concentration of the tested sample $(10 \mathrm{~g} / \mathrm{L})$.

3.4.2. Ferric Reducing Antioxidant Power (FRAP), Cupric Reducing Antioxidant Capacity (CUPRAC), $\mathrm{ABTS}^{+}$Scavenging Activity and Xanthine Oxidase Inhibition Test

FRAP and CUPRAC assays were conducted as indicated in our previous work, where these protocols were applied to measure the antioxidant properties of oligoesculin [15]. The results were expressed as the average value of two replicates. The ABTS assay was carried out to measure the potential of samples $\left(1 \mathrm{~g} / \mathrm{L}\right.$ solutions) to scavenge $\mathrm{ABTS}^{+}$radical. This test was performed according to the method proposed by Re et al. [33] but was modified by increasing the incubation time, from $6 \mathrm{~min}$ up to $45 \mathrm{~min}$ based on previous results obtained by Boẑiĉ et al. [37], which showed that caffeic acid and gallic acid oligomers increased their scavenging activity over time, reaching values even higher than those obtained for monomer molecules. For FRAP, CUPRAC, and ABTS assays, samples were solubilized in methanol with $15 \%(v / v)$ DMSO.

In order to measure the xanthine oxidase inhibitory activity, the spectrophotometric protocol reported by Chebil et al. [12] was followed with the modifications detailed by Muñiz-Mouro et al. [15].

These protocols were performed using a Shimadzu UV-1800 spectrophotometer (Shimadzu Europa GmbH, Duisburg, Germany).

\subsubsection{Assays for the Determination of Phenolic Compounds}

Phenolic content was determined using two different protocols: Folin-Ciocalteu assay [38] and 4-aminoantipyrine (4-AAP) assay [39]. The Folin-Ciocalteu protocol was performed with the modifications indicated by Muñiz-Mouro et al. [15], and the values were obtained as the average of duplicates. The percentage of reducing phenolic compounds (RPC) present in each product was calculated in relation to its control. 
In the 4-AAP assay, $920 \mu \mathrm{L}$ of diluted samples or phenol standards (from 0.1 to $4.5 \mathrm{mg} / \mathrm{L}$ ) were added to $50 \mu \mathrm{L}$ of sodium-potassium tartrate buffer ( $\mathrm{pH}$ 9.5), $50 \mu \mathrm{L}$ of 4-AAP $(20 \mathrm{~g} / \mathrm{L})$, and $50 \mu \mathrm{L}$ of potassium ferricyanide $(20 \mathrm{~g} / \mathrm{L})$. After $5 \mathrm{~min}$ of incubation, an absorbance at $510 \mathrm{~nm}$ was recorded in a BioTek PowerWave XS2 microplate spectrophotometer (Biotek Germany, Friedrichshall, Germany). The results were expressed in $\mathrm{mg}$ of phenol equivalents per $\mathrm{g}$ of freeze-dried sample as the average of four replicates. The phenolic content of each product was calculated in relation to its control.

\subsubsection{Structural Analysis: MALDI-TOF and HPSEC}

Absolute masses were determined by MALDI-TOF based on the protocol described by Anthoni et al. [30], using the equipment described in our previous work [15] and using DHB matrix but choosing the positive ionization and linear operation modes. The lyophilized samples were dissolved $(1 \mathrm{~g} / \mathrm{L})$ in acetonitrile/water $(30: 70, v / v)$ with $0.1 \%$ TFA.

The average molecular weight $(\mathrm{Mw})$ of oligorutin fractions $(3 \mathrm{~g} / \mathrm{L}$ solutions) was analyzed by high-performance size exclusion chromatography (HPSEC) using the method described by Dávila et al. [31] with some modifications: the injection volume was switched to $40 \mu \mathrm{L}$, the flow rate to $0.4 \mathrm{~mL} / \mathrm{min}$, and the temperature of $50{ }^{\circ} \mathrm{C}$. This technique also made it possible to determine the number average $(\mathrm{Mn})$ and the degree of polydispersity $(\mathrm{Mw} / \mathrm{Mn})$. The degree of polymerization (DP) was calculated as the ratio between Mw and the molecular mass of rutin. The chromatograms obtained were processed using the software ChromNAV 2.0 HPLC Software version 1.11.02 (JASCO, Easton, MD, USA).

\subsubsection{Production of Oligomers with Similar Molecular Mass}

In order to assess whether the properties of the rutin oligomers depend solely on its molecular weight, regardless of the enzyme activity used during oligomerization, reactions A and B were repeated following the same experimental procedure explained in Section 3.3, and the product medium obtained was ultrafiltrated into five different fractions instead of three. In addition, to avoid different protein contents associated to the use of different enzyme activities, Experiment A was supplemented with thermally inactivated laccase until the amount of enzyme was equivalent to that used in Experiment $B$. The fractions obtained after ultrafiltration were: F5', retentate of a $30 \mathrm{kDa}$ membrane, F4', permeate of 30 and retentate of $10 \mathrm{kDa}$ membranes, $\mathrm{F}^{\prime}$, permeate of 10 and retentate of $3 \mathrm{kDa}$ membranes, F2', permeate of 3 and retentate of $1 \mathrm{kDa}$ membranes, and F1', permeate of $1 \mathrm{kDa}$ membrane. The molecular weights of all lyophilized fractions were determined by HPSEC and similar products of both reactions were selected to assess their antioxidant properties by FRAP and CUPRAC assays.

\subsubsection{Thermal Stability Study of Rutin Oligomers}

The thermal properties of the oligorutins produced were evaluated by thermogravimetric analysis (TGA). This analysis was performed on a Mettler Toledo TGA/DSC1 thermobalance (Mettler-Toledo, Columbus, $\mathrm{OH}, \mathrm{USA}$ ), with a heating rate at $10{ }^{\circ} \mathrm{C} \cdot \mathrm{min}^{-1}$ from 25 to $600{ }^{\circ} \mathrm{C}$ under a nitrogen flow $\left(20 \mathrm{~mL} \cdot \mathrm{min}^{-1}\right)$, placing approximately $2-4 \mathrm{mg}$ of lyophilized samples in an aluminum crucible. For each sample, the decomposition intervals and the maximum decomposition temperature rate (MRDT) were obtained, as well as the final percentage of solids remaining at $600{ }^{\circ} \mathrm{C}$.

\section{Conclusions}

The effect of the starting enzymatic activity in the laccase-mediated oxidative oligomerization of rutin was studied using the natural enzyme from T. versicolor in a food-compatible reaction medium. The rutin oligomers with the best characteristics for its use as a nutraceutical were obtained in the reaction with the lowest laccase activity $(1000 \mathrm{U} / \mathrm{L})$. The rutin oligomeric AF3 fraction obtained with this enzyme dosage significantly improved the apparent aqueous solubility and xanthine oxidase inhibitory activity compared to its control reaction, without compromising the antioxidant activity. The thermal stability of rutin oligomers was negatively affected by increasing the enzyme 
activity involved in the oligomerization reaction. The hypothesis of different molecular structure for rutin oligomers produced under different laccase activities was confirmed by comparing the antioxidant capacity of similar mean molecular mass oligomers produced involving different laccase activities, leading to the conclusion that higher enzyme dosages promoted the formation of multiple intermolecular bonds between rutin units, which negatively affected their antioxidant activity.

To our knowledge, this is the first study that focuses on the effect of laccase activity upon the products obtained in enzymatic oligomerization of the rutin flavonoid as a key parameter to enhance and tailor their physicochemical and biological properties. Further experiments will be required to optimize and scale up the production of this rutin oligomer, to evaluate its bioactive properties more exhaustively, and to provide more insight on the chemical structure of rutin oligomers.

Supplementary Materials: The following are available online at http:/ /www.mdpi.com/2073-4344/8/8/321/s1, Figure S1. Solubility study of rutin in ethanol:acetate buffer mixtures (0.1 M, pH 5), Figure S2. Chemical structure of rutin. Rutin monomer has two para-substituted and two non-para-substituted phenolic groups, one of each type bonded to the A ring and the other to the B ring, Figure S3. MALDI-TOF analysis of rutin oligomer fractions produced in reactions A and B, Figure S4. HPSEC chromatograms for laccase and rutin. Mp: peak molecular weight, tR: retention time, Figure S5. HPSEC chromatograms for rutin oligomer fractions AF5' and BF4', Table S1. Total flavonoid content (TFC) in $10 \mathrm{~g} / \mathrm{L}$ solutions of the different oligomer fractions in $50 \% \mathrm{MeOH}: \mathrm{H} 2 \mathrm{O}$ and in supernatants of oversaturated $(10 \mathrm{~g} / \mathrm{L})$ aqueous solutions, Table S2. MALDI-TOF results of the different fractions of rutin oligomers produced in reaction A and suggested compounds, detected as the deprotonated compounds, Table S3. MALDI-TOF results of the different fractions of rutin oligomers produced in reaction B and suggested compounds, detected as the deprotonated compounds, Table S4. TGA main parameters for high molecular mass rutin oligomers obtained in reactions A and B, compared to control experiments, Table S5. Summary of tested oligorutin properties.

Author Contributions: G.E., B.G. and J.M.L. conceived and designed the experiments; A.M.-M. performed all the experiments; A.M.-M., G.E., B.G., M.T.M. and T.A.L.-C. analyzed the data; all authors contributed to the writing of the paper.

Funding: This work was financially supported by the Spanish Ministry of Economy and Competitiveness (CTQ2014-58879-JIN). The authors belong to the Galician Competitive Research Group GRC-ED431C 2017/29 and to the strategic group CRETUS (AGRUP2015/02). All these programs are co-funded by FEDER. B. Gullón thanks the Spanish Ministry of Economy and Competitiveness for her postdoctoral fellowship (Grant reference IJCI-2015-25305).

Acknowledgments: The authors would like to acknowledge the use of RIAIDT-USC analytical facilities.

Conflicts of Interest: The authors declare no conflict of interest.

\section{References}

1. Romano, B.; Pagano, E.; Montanaro, V.; Fortunato, A.L.; Milic, N.; Borrelli, F. Novel insights into the pharmacology of flavonoids. Phyther. Res. 2013, 27, 1588-1596. [CrossRef] [PubMed]

2. Rice-Evans, C.A.; Miller, N.J.; Paganga, G. Structure-antioxidant activity relationships of flavonoids and phenolic acids. Free Radic. Biol. Med. 1996, 20, 933-956. [CrossRef]

3. Borges Bubols, G.; da Rocha Vianna, D.; Medina-Remón, A.; von Poser, G.; Lamuela-Raventos, R.M.; Eifler-Lima, V.L.; Cristina Garcia, S. The Antioxidant Activity of Coumarins and Flavonoids. Mini-Rev. Med. Chem. 2013, 13, 318-334. [CrossRef]

4. Hosseinzadeh, H.; Nassiri-Asl, M. Review of the protective effects of rutin on the metabolic function as an important dietary flavonoid. J. Endocrinol. Investig. 2014, 37, 783-788. [CrossRef] [PubMed]

5. Scalbert, A.; Manach, C.; Morand, C.; Rémésy, C.; Jiménez, L. Dietary Polyphenols and the Prevention of Diseases. Crit. Rev. Food Sci. Nutr. 2005, 45, 287-306. [CrossRef] [PubMed]

6. Rufa, L.; Yunning, Z.; Rui, W.; Jianying, L. A Study on the Extract of Tartary Buckwheat I. Toxicological Safety of the Extract of Tartary Buckwheat. Small 2001, 21, 602-607.

7. Gullón, B.; Lú-Chau, T.A.; Moreira, M.T.; Lema, J.M.; Eibes, G. Rutin: A review on extraction, identification and purification methods, biological activities and approaches to enhance its bioavailability. Trends Food Sci. Technol. 2017, 67, 220-235. [CrossRef]

8. NIH Dietary Supplement Label Database. Available online: https://www.dsld.nlm.nih.gov/dsld/index.jsp (accessed on 10 April 2018).

9. Krewson, B.C.F.; Naghskit, J. Some Physical Properties of Rutin. J. Pharm. Sci. 1952, 41, 582-587. [CrossRef] 
10. Rothwell, J.A.; Day, A.J.; Morgan, M.R.A. Experimental Determination of Octanol-Water Partition Coefficients of Quercetin and Related Flavonoids. J. Agric. Food Chem. 2005, 53, 4355-4360. [CrossRef] [PubMed]

11. Paczkowska, M.; Mizera, M.; Piotrowska, H.; Szymanowska-Powałowska, D.; Lewandowska, K.; Goscianska, J.; Pietrzak, R.; Bednarski, W.; Majka, Z.; Cielecka-Piontek, J. Complex of rutin with $\beta$-Cyclodextrin as potential delivery system. PLoS ONE 2015, 10, e0120858. [CrossRef] [PubMed]

12. Chebil, L.; Rhouma, G.B.; Chekir-Ghedira, L.; Ghoul, M. Enzymatic Polymerization of Rutin and Esculin and Evaluation of the Antioxidant Capacity of Polyrutin and Polyesculin. In Biotechnology; Ekinci, D., Ed.; InTech: London, UK, 2015; pp. 117-133, ISBN 978-953-51-2040-7.

13. Chung, J.E.; Kurisawa, M.; Kim, Y.J.; Uyama, H.; Kobayashi, S. Amplification of antioxidant activity of catechin by polycondensation with acetaldehyde. Biomacromolecules 2004, 5, 113-118. [CrossRef] [PubMed]

14. Kurisawa, M.; Chung, J.E.; Uyama, H.; Kobayashi, S. Enzymatic synthesis and antioxidant properties of poly(rutin). Biomacromolecules 2003, 4, 1394-1399. [CrossRef] [PubMed]

15. Muñiz-Mouro, A.; Oliveira, I.M.; Gullón, B.; Lú-Chau, T.A.; Moreira, M.T.; Lema, J.M.; Eibes, G. Comprehensive investigation of the enzymatic oligomerization of esculin by laccase in ethanol:water mixtures. RSC Adv. 2017, 7, 38424-38433. [CrossRef]

16. Ghoul, M.; Chebil, L. Enzymatic polymerization of phenolic compounds by oxidoreductases. In SpringerBriefs in Molecular Science; Springer: Dordrecht, The Netherlands, 2012; ISBN 978-94-007-3918-5.

17. Jeon, J.R.; Baldrian, P.; Murugesan, K.; Chang, Y.S. Laccase-catalysed oxidations of naturally occurring phenols: From in vivo biosynthetic pathways to green synthetic applications. Microb. Biotechnol. 2012, 5, 318-332. [CrossRef] [PubMed]

18. Hollmann, F.; Arends, I.W.C.E. Enzyme Initiated Radical Polymerizations. Polymers 2012, 4, $759-793$. [CrossRef]

19. Anthoni, J.; Lionneton, F.; Wieruszeski, J.M.; Magdalou, J.; Engasser, J.M.; Chebil, L.; Humeau, C.; Ghoul, M. Investigation of enzymatic oligomerization of rutin. Rasayan J. Chem. 2008, 1, 718-731.

20. Rhouma, G.B.; Chebil, L.; Mustapha, N.; Krifa, M.; Ghedira, K.; Ghoul, M.; Chékir-Ghédira, L. Cytotoxic, genotoxic and antigenotoxic potencies of oligorutins. Hum. Exp. Toxicol. 2013, 32, 881-889. [CrossRef] [PubMed]

21. Sun, X.; Bai, R.; Zhang, Y.; Wang, Q.; Fan, X.; Yuan, J.; Cui, L.; Wang, P. Laccase-Catalyzed Oxidative Polymerization of Phenolic Compounds. Appl. Biochem. Biotechnol. 2013, 171, 1673-1680. [CrossRef] [PubMed]

22. Burda, S.; Oleszek, W. Antioxidant and Antiradical Activities of Flavonoids. J. Agric. Food Chem. 2001, 49, 2774-2779. [CrossRef] [PubMed]

23. Heim, K.E.; Tagliaferro, A.R.; Bobilya, D.J. Flavonoid antioxidants: Chemistry, metabolism and structure-activity relationships. J. Nutr. Biochem. 2002, 13, 572-584. [CrossRef]

24. Cos, P.; Ying, L.; Calomme, M.; Hu, J.P.; Cimanga, K.; Van Poel, B.; Pieters, L.; Vlietinck, A.J.; Vanden Berghe, D. Structure-activity relationship and classification of flavonoids as inhibitors of xanthine oxidase and superoxide scavengers. J. Nat. Prod. 1998, 61, 71-76. [CrossRef] [PubMed]

25. Kumar, R.; Darpan; Sharma, S.; Singh, R. Xanthine oxidase inhibitors: A patent survey. Expert Opin. Ther. Pat. 2011, 21, 1071-1108. [CrossRef] [PubMed]

26. Hayashi, T.; Kazuko, S.; Masaru, K.; Munehisa, A.; Mineo, S.; Naokata, M. Inhibition of Cow's Milk Xanthine Oxidase by Flavonoids. J. Nat. Prod. 1988, 51, 345-348. [CrossRef] [PubMed]

27. Zhao, C.; Song, J.F.; Zhang, J.C. Determination of total phenols in environmental wastewater by flow-injection analysis with a biamperometric detector. Anal. Bioanal. Chem. 2002, 374, 498-504. [CrossRef] [PubMed]

28. Méchin, V.; Baumberger, S.; Pollet, B.; Lapierre, C. Peroxidase activity can dictate the in vitro lignin dehydrogenative polymer structure. Phytochemistry 2007, 68, 571-579. [CrossRef] [PubMed]

29. Chaaban, H.; Ioannou, I.; Chebil, L.; Slimane, M.; Gérardin, C.; Paris, C.; Charbonnel, C.; Chekir, L.; Ghoul, M. Effect of heat processing on thermal stability and antioxidant activity of six flavonoids. J. Food Process. Preserv. 2017, 41, e13203. [CrossRef]

30. Anthoni, J.; Chebil, L.; Lionneton, F.; Magdalou, J.; Humeau, C.; Ghoul, M. Automated analysis of synthesized oligorutin and oligoesculin by laccase. Can. J. Chem. 2011, 89, 964-970. [CrossRef] 
31. Dávila, I.; Gullón, P.; Andrés, M.A.; Labidi, J. Coproduction of lignin and glucose from vine shoots by eco-friendly strategies: Toward the development of an integrated biorefinery. Bioresour. Technol. 2017, 244, 328-337. [CrossRef] [PubMed]

32. Zimmermann, Y.-S.; Shahgaldian, P.; Corvini, P.F.X.; Hommes, G. Sorption-assisted surface conjugation: A way to stabilize laccase enzyme. Appl. Microbiol. Biotechnol. 2011, 92, 169-178. [CrossRef] [PubMed]

33. Re, R.; Pellegrini, N.; Proteggente, A.; Pannala, A.; Yang, M.; Rice-Evans, C. Antioxidant activity applying an improved ABTS radical cation decolorization assay. Free Radic. Biol. Med. 1999, 26, 1231-1237. [CrossRef]

34. Wariishi, H.; Valli, K.; Gold, M.H. Manganese(II) oxidation by manganese peroxidase from the basidiomycete Phanerochaete chrysosporium: Kinetic mechanism and role of chelators. J. Biol. Chem. 1992, 267, 23688-23695. [PubMed]

35. Kim, D.-O.; Jeong, S.W.; Lee, C.Y. Antioxidant capacity of phenolic phytochemicals from various cultivars of plums. Food Chem. 2003, 81, 321-326. [CrossRef]

36. Gullón, B.; Gullón, P.; Lú-Chau, T.A.; Moreira, M.T.; Lema, J.M.; Eibes, G. Optimization of solvent extraction of antioxidants from Eucalyptus globulus leaves by response surface methodology: Characterization and assessment of their bioactive properties. Ind. Crops Prod. 2017, 108, 649-659. [CrossRef]

37. Boẑiĉ, M.; Gorgieva, S.; Kokol, V. Laccase-mediated functionalization of chitosan by caffeic and gallic acids for modulating antioxidant and antimicrobial properties. Carbohydr. Polym. 2012, 87, 2388-2398. [CrossRef]

38. Singleton, V.L.; Rossi, J.A., Jr. Colorimetry of Total Phenolics with Phosphomolybdic-Phosphotungstic Acid Reagents. Am. J. Enol. Vitic. 1965, 16, 144-158. [CrossRef]

39. EPA, U.S. Phenolics (Spectrophotometric, Manual 4-AAP with Distillation). Available online: https://www. epa.gov/hw-sw846/sw-846-test-method-9065-phenolics-spectrophotometric-manual-4-aap-distillation (accessed on 4 August 2018).

(C) 2018 by the authors. Licensee MDPI, Basel, Switzerland. This article is an open access article distributed under the terms and conditions of the Creative Commons Attribution (CC BY) license (http:/ / creativecommons.org/licenses/by/4.0/). 